\title{
Feasibility evaluation of amide proton transfer-weighted imaging in the parotid glands: a strategy to recognize artifacts and measure APT value
}

\author{
Yu Chen ${ }^{1 \#}$, Xiaoqi Wang ${ }^{2 \#}$, Tong Su ${ }^{1 \#}$, Zhentan $X u^{1}$, Yunting Wang ${ }^{3}$, Zhuhua Zhang ${ }^{1}$, Huadan Xue ${ }^{1}$, \\ Zhizheng Zhuo ${ }^{2}$, Yuanli Zhu', Zhengyu Jin ${ }^{1 *}$, Tao Zhang ${ }^{3 *}$ \\ ${ }^{1}$ Department of Radiology, Peking Union Medical College Hospital, Chinese Academy of Medical Sciences, Beijing, China; ${ }^{2}$ Philips Healthcare, \\ Beijing, China; ${ }^{3}$ Department of Stomatology, Peking Union Medical College Hospital, Chinese Academy of Medical Sciences, Beijing, China
}

\#These authors contributed equally to this work and are co-first authors.

*These authors contributed equally to this work.

Correspondence to: Zhengyu Jin. Department of Radiology, Peking Union Medical College Hospital, Chinese Academy of Medical Sciences, No. 1 Shuai Fu Yuan, Dong Cheng District, Beijing 100730, China. Email: jinzy@pumch.cn; Tao Zhang. Department of Stomatology, Peking Union Medical College Hospital, Chinese Academy of Medical Sciences, No. 1 Shuai Fu Yuan, Dong Cheng District, Beijing 100730, China. Email: drtzhang@126.com.

Background: The feasibility and image quality of three-dimensional (3D) amide proton transfer (APT)weighted (APTw) in parotid tumor lesions have not been well established in previous studies. This study aimed to evaluate the utility of APT imaging in parotid lesions and glands.

Methods: Patients with parotid lesions received 3D turbo spin echo (TSE) APTw on a 3.0T scanner. Two radiologists, who were blinded to the clinical data, independently evaluated the APTw image quality using 4-point Likert scales ( $1=$ poor, $4=$ excellent) in terms of integrity and hyperintensity artifacts. An image quality selection protocol was built based on the two scores. Evaluable images (integrity score $>1$ ) and trustable images (integrity score $>3$ and hyperintensity artifacts score $>2$ ) were then enrolled for APTw value comparison between parotid lesions and glands.

Results: Forty consecutive patients were included in this study. Four patients were excluded due to severe motion $(n=3)$ or dental $(n=1)$ artifacts, and 36 patients received the APT sequence. Among these, more parotid tumor lesions $(34 / 36,94.4 \%)$ than normal parotid glands $(23 / 31,74.2 \%)$ revealed excellent integrity scores $($ score $=4)(\mathrm{P}=0.034)$. Most parotid tumor lesions $(24 / 34,70.6 \%)$ and glands $(16 / 28,57.1 \%)$ revealed no or little hyperintensity artifacts for diagnosis (scores 3 and 4). APT values of parotid lesions and glands in the evaluable groups were $2.11 \% \pm 1.15 \%$ and $1.60 \% \pm 1.56 \%$, respectively, and the difference was not significant $(\mathrm{P}=0.197)$. APT values of parotid lesions and glands in the trustable groups were $1.99 \% \pm 1.18 \%$ and $1.03 \% \pm 1.09 \%$, respectively, and the difference was statistically significant $(\mathrm{P}=0.018)$.

Conclusions: 3D APTw could be used to differentiate parotid tumors and normal parotid glands; however, the technology still needs to be improved to remove artifacts. In our study, most APTw images of tumor lesions in parotid glands had acceptable image quality, and these APTw images are feasible for diagnostic use.

Keywords: Parotid gland; magnetic resonance imaging (MRI); amide proton transfer (APT); image quality

Submitted May 20, 2020. Accepted for publication Mar 18, 2021.

doi: $10.21037 /$ qims-20-675

View this article at: http://dx.doi.org/10.21037/qims-20-675 


\section{Introduction}

Preoperative fine-needle aspiration biopsy results can provide important information on salivary gland mass lesions, such as whether the lesion is inflammatory or neoplastic and lymphoid or epithelial (1). However, sampling errors in heterogeneous tumors and nondiagnostic aspirate due to insufficient biopsy coverage occur in more than $10 \%$ of salivary gland tumors (2).

Modern imaging plays a key role in the differentiation of benign and malignant parotid lesions and selecting appropriate surgical strategies or radiotherapy (3). Highresolution ultrasonography, computed tomography (CT), and conventional magnetic resonance imaging (MRI) are the main imaging methods. MRI is superior in displaying the lesion interface and surrounding tissues without ionizing radiation. In the past, the roles of functional MRI, such as diffusion-weighted imaging (DWI) and dynamic contrastenhanced (DCE) MRI, have been evaluated in parotid lesions (4-7). However, it is still challenging to differentiate benign and malignant lesions based on functional MRI. Therefore, advanced MRI techniques have been explored to provide biomarkers for the qualitative diagnosis of parotid lesions (8).

Amide proton transfer (APT) imaging is a non-contrast molecular imaging method. APT scopes the concentration of amide protons in tissue proteins and peptides by selectively saturating amide protons magnetization at 3.5 parts-per-million (ppm) and observing the affected water MR signal due to exchange between saturated amide protons and water protons. The much larger pool size of endogenous water protons (compared to the amide protons) and the continuous chemical exchange process has enabled the detection of free proteins and peptides with extremely low concentrations $(9,10)$. APT MRI is a potential tool for tumor detection and characterization in the brain (11). More sophisticated clinical chemical exchange saturation transfer (CEST) applications exist that show a correlation between APT with therapy response (12-14) and patient outcome/survival (15).

Investigators in recent human studies have also reported preliminary APT findings in the breast (16-19), cervices $(20,21)$, prostate $(22,23)$, and chest $(8)$, demonstrating that it is an effective tool for tumor detection and characterization. APT values were higher in cancers than in normal surrounding tissues (22) and benign tumors (8). However, investigations applying the APT method in parotid tumors and normal parotid glands have not been well established in previous literature. There were a few reports about APT studies of parotid glands. Jing Yuan et al. (11) demonstrated that parotid glands showed the highest APTw signal ( $7.6 \%$ on average), while the APTw signals in other tissues were relatively moderate. Also, Yun Jung Bae et al. (24) demonstrated that the APTw signal was significantly higher in malignant parotid tumors than benign tumors $(\mathrm{P}<0.001)$. The APTw-signals exhibited an excellent area under the curve for predicting malignant tumors, which were markedly higher than the combined use of DWI and DWI-MRI ( $\mathrm{P}=0.021$ and 0.028 , respectively). These studies used two-dimensional (2D) APT sequences, and only one slice was scanned for APTw. With a 2D readout, the shorter acquisition time is preferred in clinical settings, with only one single-slice acquisition. However, a three-dimensional (3D) readout could cover the whole lesion with multi-slice imaging and minimize relaxation loss between slices (25). Moreover, the commercially available 3D APT sequence for clinical use is easier for access and evaluation. Also, the 3D APT sequence is faster than the $3 \mathrm{D}$ version for five slices.

We expected APT MRI to provide head and neck image values to differentiate malignant tumors from benign lesions and normal tissues $(11,26)$. Our first impression of APT application in parotid glands was unsatisfying because of relatively frequent image artifacts. However, a portion of parotid tumor APTw images showed correct diagnosis (24). Therefore, in this study, we investigated the feasibility of APTw imaging application in parotid glands and lesions in terms of image integrity and quality, and evaluated its ability to differentiate between lesions and normal tissues.

\section{Methods}

\section{Participants}

This study was conducted following the Declaration of Helsinki and was approved by an institutional review board. Written informed consent was obtained from the patients. Between December 2017 and October 2018, 40 consecutive patients with lesions in the parotid glands who planned to receive MR examinations were enrolled into this study. Four patients were excluded because the anatomic planning images were degraded by severe motion $(n=3)$ or dental $(n=1)$ artifacts. In total, 36 patients underwent 3D turbo-spin-echo (TSE) APTw MR imaging on a 3T MR scanner (Ingenia CX, Philips Healthcare, the Netherlands) equipped with dual radiofrequency (RF) transmit coils and a 32-channel head-coil for image acquisition. The patient selection flowchart is shown in Figure 1. 


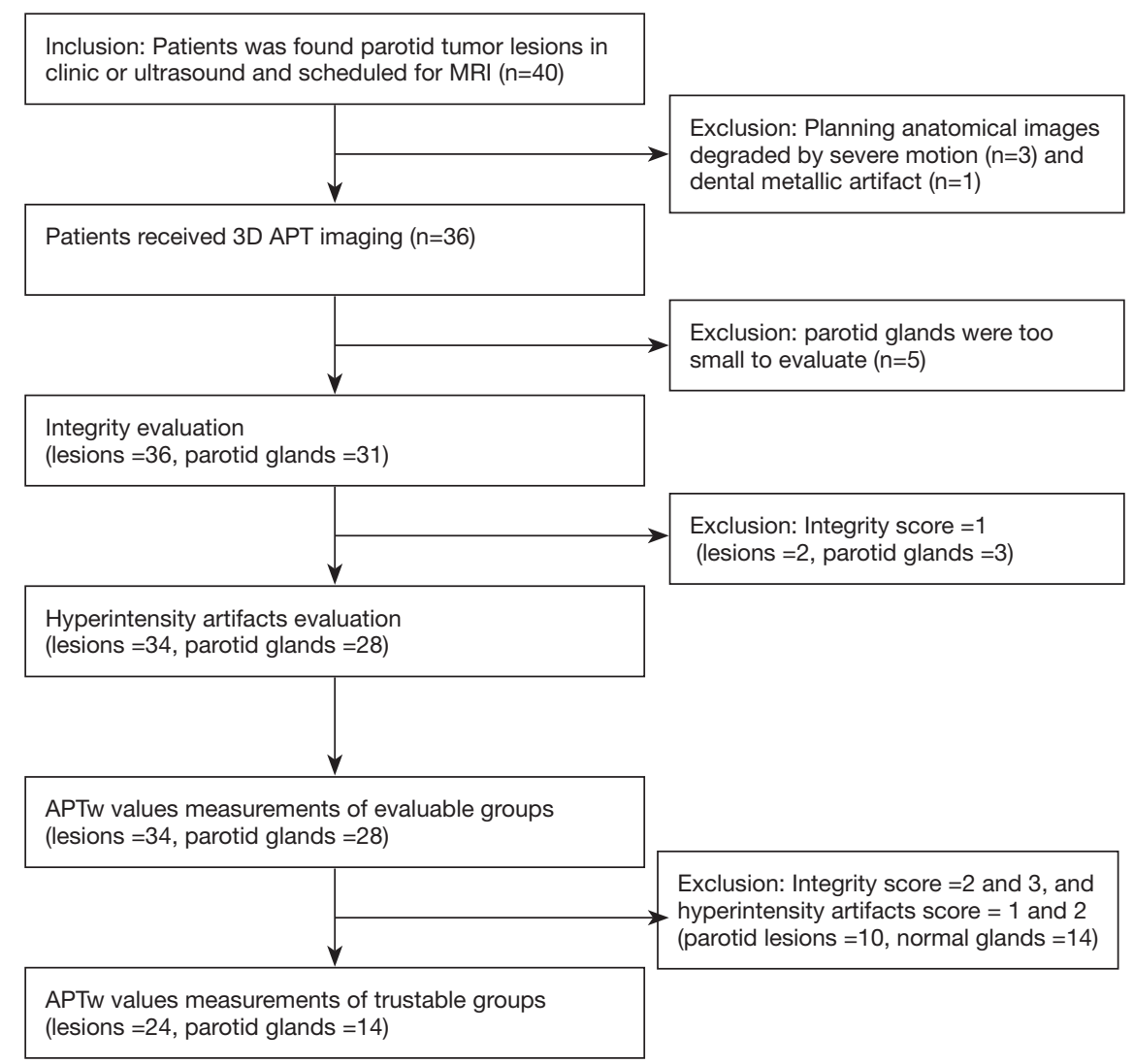

Figure 1 Patient selection and APT evaluation flowchart. APT, amide proton transfer.

\section{MRI}

All patients underwent MR imaging with a clinical 3.0-Testla MR system (Ingenia CX, Philips Healthcare, Best, the Netherlands) with a body coil for radiofrequency transmission and a 32-channel coil for signal reception.

Routine clinical imaging protocols were performed before the APT sequence, including axial T1 weighted turbo spin echo (T1w-TSE) [repetition time (TR), 701 $\mathrm{ms}$; (echo time) TE, $14.772 \mathrm{~ms}$; number of signal averaged (NSA), 2; slice thickness, $4 \mathrm{~mm}$; matrix, $512 \times 512$ ], and axial T2 weighted turbo spin echo (T2w-TSE) (TR, 4,679 ms; TE, $114.912 \mathrm{~ms}$; NSA, 2; slice thickness, $4 \mathrm{~mm}$; matrix, $512 \times 512)$. The anatomical images were also used to plan the field-of-view for the APT sequence with better localization.

The commercially available 3D APT sequence embedded the 3D TSE sequences. The 3D APT sequence parameters were as follows: slices, five; flip angle $(\mathrm{FA})=90 ; \mathrm{TR} / \mathrm{TE}$ $=2,000 / 8$, the field of view, $250 \times 250 \mathrm{~mm}^{2}$; slice thickness, $5 \mathrm{~mm}$; voxel size, $2.50 \times 2.53 \times 5.00 \mathrm{~mm}^{2}$; TSE factor, 158 ; and Sensitivity Encoding (SENSE) factor, 1.6. The scan time of this sequence was 2 minutes and 40 seconds. Continuous RF saturation with no gap is essential for APT labeling. The MR scanner was equipped with two sets of transmitting RF amplifiers and coils, and the two transmission channels were turned on alternatively to produce four saturation pulses, each $500 \mathrm{~ms}$ in duration with an amplitude of $2 \mu \mathrm{T}$, to achieve a sufficient saturation of 2 seconds. The z-spectrum, with the saturation pre-pulse targeting at $3.5 \mathrm{ppm}, 3.5 \pm 0.8 \mathrm{ppm}$, and $-3.5 \mathrm{ppm},-3.5 \pm 0.8 \mathrm{ppm}$ and $-1,560 \mathrm{ppm}$ (according to the water proton frequency), was measured for each voxel. Images with saturation at the $+3.5 \mathrm{ppm}$ were acquired three times with an echo shift of 0.5 msec sequentially to calculate B0 field mapping $(27,28)$ and then for z-spectrum correction (29).

APTw value calculations were performed in the MR control console, according to previously published algorithms (29). B0 homogeneity was firstly measured and calculated via the echo shift in TSE acquisitions. With saturation at each offset, signals would be shifted to the corrected offsets in the z-spectrum based on the 

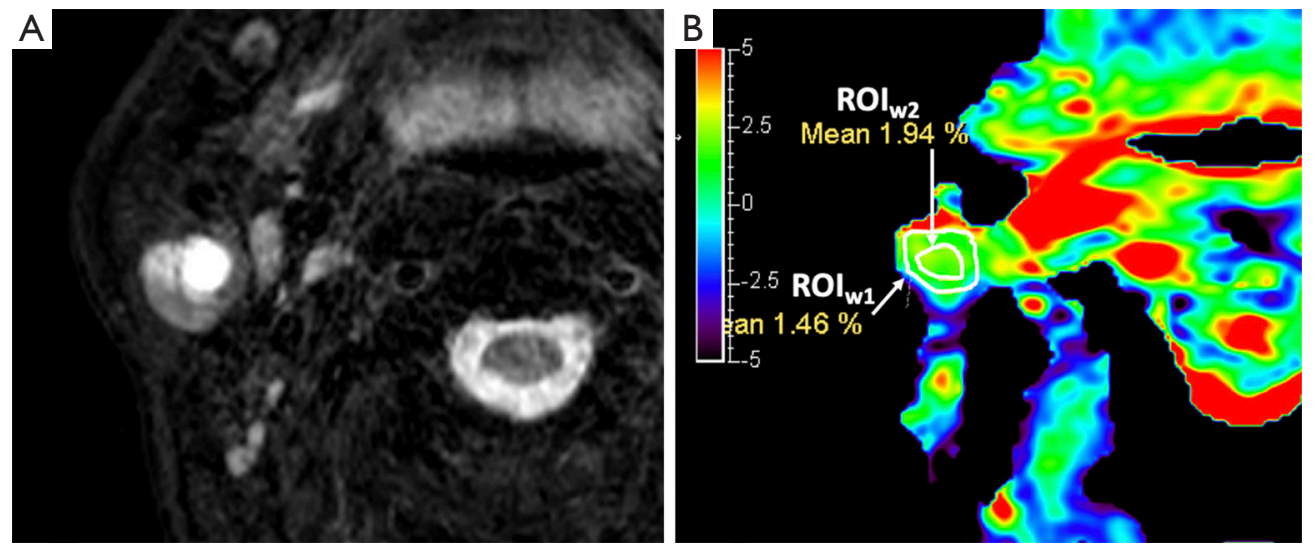

Figure 2 An APTw image example with excellent lesion image quality. A round lesion was found in the right parotid gland of a 65 -yearold male, as shown on T2WI (A). The corresponding APTw image of the same slice is shown in (B). The image quality for this APTw map in terms of integrity and hyperintensity artifacts on the lesion was scored as 4 and 4 , respectively. The lesion was considered as being in the trustable group. The average APTw value of the lesion was $1.94 \%$ for the $\mathrm{ROI}_{\mathrm{w} 2}$, drawn avoiding surrounding hyperintensity artifacts in the $\mathrm{ROI}_{\mathrm{w} 1}$. The arrows mean APTw values in ROI.

B0 field map. Signals at targeting offset $S(-3.5 \mathrm{ppm})_{\text {corrected }}$ and $S(+3.5 \mathrm{ppm})_{\text {corrected }}$ were calculated via the Lagrange interpolation, using the measured signals and the relative distances of measured offsets to targeting offsets.

APT weighting, measured as Magnetization Transfer Ratio (MTR) asymmetry, which is the signal difference between the corrected $\pm 3.5 \mathrm{ppm}$ images normalized to the $-1,560$ ppm image, was calculated according to the following equation:

$$
A P T \text { weighting }=\frac{S(-3.5 p p m)_{\text {corrected }}-S(+3.5 p p m)_{\text {corrected }}}{S(-1560 \mathrm{ppm})}
$$

\section{Image analysis}

MR images and data were transferred to a workstation (Intellispace Portal; v. 10.1.0.64190; Philips Healthcare) for further analysis. One radiologist (YC) and one radiology scientist $(\mathrm{XQW})$ with 10 years of head and neck experience independently reviewed all MR images. They were blinded to all patients' clinical and histopathological data. Both the tumor lesions and parotid glands without lesions (from now on referred to as the parotid glands) were evaluated on APTw images, and both readers obtained consensus results.

The patients' sex, age, and largest lesion diameter in axial images were recorded. The locations of lesions in the parotid gland were divided into upper, middle, and lower parts.

\section{Qualitative analysis}

Qualitative analysis was applied first to identify artifacts and evaluate image quality. We found strong artifacts at regions with large B0 inhomogeneity near air cavities and bone, where extrapolation fitting (rather than interpolation) had to be used for z-spectrum correction. Also, the low pass filter in APTw calculation spreads the extreme APTw values $(>5 \%$ and $<-5 \%)$ at the near skull and ear to parotid glands and lesions, which is our region of interest (ROI). In our study, we found that some APTw images of parotid glands and lesions were unsatisfactory for diagnosis due to these hyperintensity artifacts (APTw value $>5 \%$ ) or signal loss areas (APTw value $<-5 \%$ ). Hence, we need to recognize these image artifacts and decide if the samples could be enrolled in APTw value quantification.

The radiologists evaluated the parotid glands and lesions and drew the region of interest $\left(\mathrm{ROI}_{\mathrm{w1}}\right)$ on APTw mapping by manually delineating the whole tumor lesion or parotid gland concerning T2W images (Figures 2-4).

Firstly, a signal loss area of APTw images of lesions or parotid glands was evaluated within the coverage of $\mathrm{ROI}_{\mathrm{w} 1}$ (Figures 2-4), in terms of the integrity scores based on a 4-point Likert scale: $4=$ excellent, the entire lesion or normal parotid gland was displayed; $3=$ good, more than $50 \%$ of the lesion or normal parotid gland was displayed; $2=$ moderate, less than $50 \%$ of the lesion or normal parotid gland was displayed; $1=$ poor, the lesion or normal parotid 

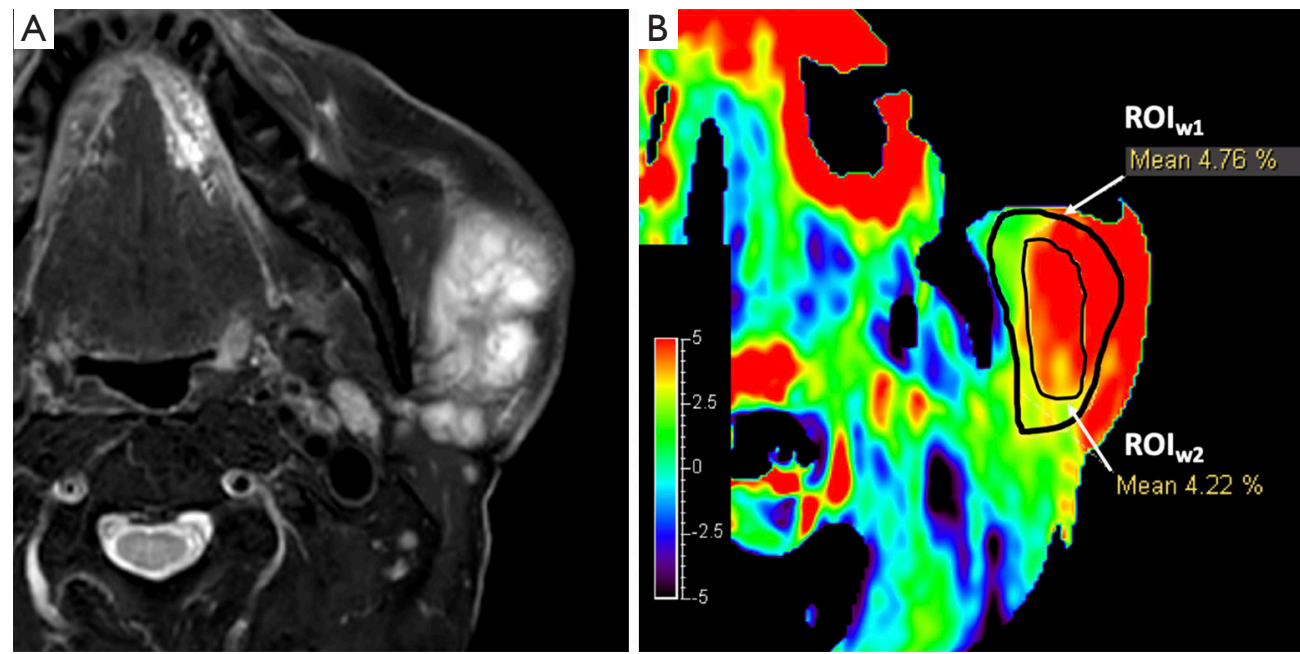

Figure 3 An APTw image example with fairly good lesion image quality. A large lesion of the left parotid gland was found in a 76-year-old male, as shown on T2WI (A). The corresponding APTw image of the same slice is shown in (B). The APT image integrity was scored as 4. The image quality in terms of hyperintensity artifacts was scored as 3, and the APT values in the ROI $\mathrm{I}_{\mathrm{1}}$ were elevated by the surrounding hyperintensity artifacts through the smoothing effects. The lesion was considered as being in the trustable group. The average 3D APTw value of the lesion was $4.22 \%$ (in $\mathrm{ROI}_{\mathrm{w} 2}$ ). The arrows mean APTw values in ROI.
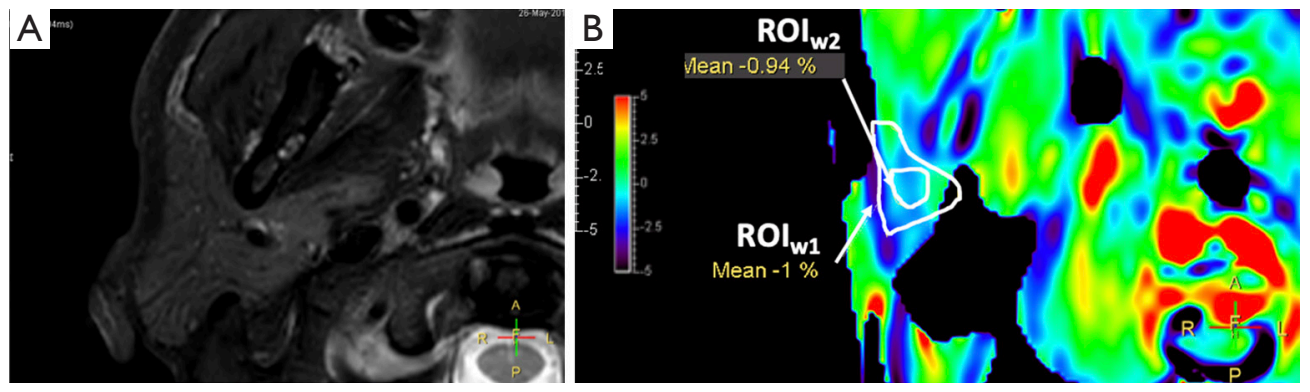

Figure 4 An APTw image example of non-optimal image quality of normal parotid gland. A 49-year-old male was found with a normal parotid gland of the right side on T2WI (A). On APTw image (B), the integrity was scored as 3, which indicates a lower confidence of the APT value (compared to Figures 2 and 3 with an integrity score 4). Although the hyperintensity artifacts scores were 4, the parotid gland image was considered as being in the evaluable group. The average 3D APTw value of the lesion was $-0.94 \%$ in $\mathrm{ROI}$ w2 The arrows mean APTw values in ROI.

gland was not displayed at all (Figure 5). The sample data was ruled out for further analysis if the integrity score was 0 .

Secondly, image quality in the $\mathrm{ROI}_{\mathrm{w} 1}$ of APTw images, in terms of the hyperintensity artifacts diffused from surrounding tissue, was then evaluated based on a 4-point Likert scale: $4=$ excellent, tumor lesion or normal parotid gland could be recognized on APTw images without or with small hyperintensity artifacts that do not impair the tumor lesion or normal parotid gland; $3=$ good, hyperintensity artifacts impair less than $50 \%$ tumor lesion or normal parotid gland; $2=$ moderate, hyperintensity artifacts impair more than $50 \%$ tumor lesion or normal parotid gland; $1=$ poor, the entire tumor lesion or normal parotid gland is impaired by hyperintensity artifacts.

Samples of the parotid gland and lesions images with an intensity score of $>1$ and a hyperintensity artifacts score $>1$ were considered evaluable groups. After removing nonoptimal image quality lesions or parotid glands (integrity score $=2$ and 3 and image quality scores $=1$ and 2), samples with intensity score $>3$ and hyperintensity artifact score $>2$ 

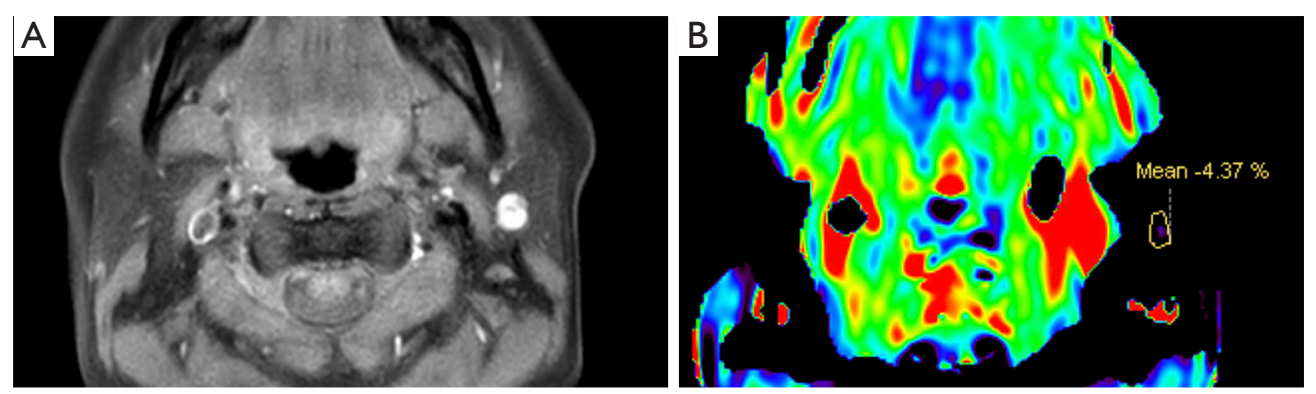

Figure 5 An APTw image example of a poor integrity parotid lesion and gland. A 45-year-old male was found with a small round lesion at the left parotid gland and a normal parotid gland of the right side on T1WI (A). On APTw image (B), both the lesion and parotid glands showed no APTw signal. Therefore, the integrity of the lesion and parotid glands were considered as score 1 and the APTw value could not be measured.

were considered as trustable groups. The corresponding APTw values would be measured and recorded to compare both the evaluable and trustable groups in the next step.

\section{Quantitative analysis}

A second ROI was drawn on the APTw images (inside the $\mathrm{ROI}_{\mathrm{w1}}$ ), avoiding the visible hyperintensity artifacts and hypointensity area, and was recorded as $\mathrm{ROI}_{\mathrm{w} 2}$. The mean APTw values in $\mathrm{ROI}_{\mathrm{w} 2}$ of tumor lesions and parotid glands were all measured (Figures 2-4). The APTw values were then compared between tumor lesions and normal parotid glands in both the evaluable and trustable groups.

\section{Statistical analysis}

Statistical analyses were performed using SPSS software (IBM SPSS, version 19.0, SPSS Inc., Chicago, IL, USA). The correlation between the locations of lesions and integrity scores/hyperintensity artifacts were analyzed by Pearson correlation. Integrity and hyperintensity artifacts scores of tumor lesions and parotid glands were compared using Fisher's exact test. The APT value between tumor lesions and parotid glands in both evaluable and trustable groups was compared using the $t$-test. All statistical tests were two-sided, and a $\mathrm{P}$ value less than 0.05 was considered statistically significant.

\section{Results}

\section{Patients' characteristics}

Among the 36 patients who received the APTw examination, there were 18 men [58 12 years (range, $33-79$ years)] and
18 women [ $45 \pm 15$ years (range, $22-73$ years)]. Data for five patients' parotid glands without lesions were excluded because the structures were too small to evaluate. Hence, there were 36 lesions and 31 parotid glands included in the analysis.

Among the 36 lesions, five lesions located were in the upper part of the parotid gland, 23 lesions were located in the middle part of the parotid gland, and eight lesions were located in the lower part of the parotid gland. The lesions' average diameter was $19.4 \pm 11.1 \mathrm{~mm}$ (range, $8.8-62.4 \mathrm{~mm}$ ). Twenty patients received surgical treatments. The pathological results were as follows: 16 benign tumors, including 6 pleomorphic adenomas (PAs); 5 Warthin tumors (WTs), including 4 basal cell adenomas (BCAs) and 1 cystadenoma (CA); and four malignant tumors (MTs), including two mucoepidermoid carcinomas, one metastasis carcinoma, and one acinar cell carcinoma. The patients' characteristics and selection flowchart is shown in Table 1 and Figure 1.

\section{Qualitative analysis}

In the integrity evaluation, 34 out of 36 lesions (94.4\%) were considered for scoring as 4 , and two lesions (5.6\%, 2/36) were not shown completely and considered score 1. Twenty-three out of 31 normal parotid glands (74.2\%) were considered for scoring 4 , four $(12.9 \%, 4 / 31)$ were score 3 , one $(3.2 \%, 1 / 31)$ was score 2 , and three $(9.7 \%, 3 / 31)$ were score 1. Parotid lesions had better APT image quality than parotid glands, and the difference between parotid tumors and parotid glands was significant $(\mathrm{P}=0.034)$.

After removing lesions or normal parotid glands with integrity scores of 1, 34 lesions and 28 parotid glands were 
Table 1 Inclusion and exclusion of patients

\begin{tabular}{|c|c|}
\hline Projects & Content \\
\hline Patient were found lesions in parotid glands & 40 \\
\hline Patients received 3D APT sequence & 36 \\
\hline Age (years) & $51.3 \pm 14.5$ \\
\hline Male numbers & 18 \\
\hline Female numbers & 18 \\
\hline Average diameter (mm) & $19.4 \pm 11.1$ \\
\hline Exclude patients & 4 \\
\hline Severe motion & 3 \\
\hline Dental metallic artifact & 1 \\
\hline \multicolumn{2}{|l|}{ Included patients } \\
\hline \multicolumn{2}{|l|}{ Lesion locations } \\
\hline Upper part of parotid glands & 5 \\
\hline Middle part of parotid glands & 23 \\
\hline Lower part of parotid glands & 8 \\
\hline Pathological results & 20 \\
\hline Benign tumors & 16 \\
\hline Pleomorphic adenomas & 6 \\
\hline Warthin tumors & 5 \\
\hline Basal cell adenomas & 4 \\
\hline Cystadenoma & 1 \\
\hline Malignant tumors & 4 \\
\hline Mucoepidermoid carcinoma & 2 \\
\hline Metastasis carcinoma & 1 \\
\hline Acinar cell carcinoma & 1 \\
\hline
\end{tabular}

evaluated regarding the hyperintensity artifacts. $17.6 \%$ (6/34), 14.7\% (5/34), 14.7\% (5/34), and 55.9\% (19/34) of parotid lesions were scored $1,2,3$, and 4 respectively; and $21.4 \%(6 / 28), 21.4 \%(6 / 28), 7.1 \%(2 / 28)$, and $50 \%$ (14/28) of normal parotid glands were scored $1,2,3$, and 4 respectively. There were no significant differences between the parotid lesions and normal parotid glands $(\mathrm{P}=0.729)$. The qualitative analysis results are shown in Table 2.

The intraclass correlation of integrity and hyperintensity artifacts scores of parotid lesions and glands by two independent readers were more than 0.9 (Table 3).

The patient selection flowchart is shown in Figure 1. Examples of parotid lesions and glands are shown in Figures 2-5. One patient (a 47-year-old male) received the B0 sequence. It was demonstrated that the region with stronger $\mathrm{B} 0$ variation, represented as the higher standard deviation in the B0 map, has more APT artifacts (Figure 6).

Table 3 The intraclass correlation of integrity and hyperintensity artifacts scores of parotid lesions and glands by two independent readers

\begin{tabular}{|c|c|c|c|}
\hline \multirow[b]{2}{*}{ Projects } & \multirow[b]{2}{*}{ ICC } & \multicolumn{2}{|c|}{ 95\% confidence interva } \\
\hline & & $\begin{array}{l}\text { Lower } \\
\text { bound }\end{array}$ & $\begin{array}{l}\text { Upper } \\
\text { bound }\end{array}$ \\
\hline \multicolumn{4}{|l|}{ Parotid lesions } \\
\hline Integrity score & 0.923 & 0.853 & 0.960 \\
\hline Hyperintensity artifacts score & 0.904 & 0.815 & 0.952 \\
\hline \multicolumn{4}{|l|}{ Parotid glands } \\
\hline Integrity score & 0.952 & 0.901 & 0.977 \\
\hline Hyperintensity artifacts score & 0.963 & 0.921 & 0.983 \\
\hline
\end{tabular}

Table 2 Qualitative analysis of parotid lesions and normal parotid glands

\begin{tabular}{|c|c|c|c|c|c|}
\hline Projects & Score $1(\%)$ & Score $2(\%)$ & Score 3 (\%) & Score 4 (\%) & $P$ value \\
\hline Integrity evaluation & & & & & 0.034 \\
\hline Parotid lesions $(n=36)$ & $2(5.6)$ & 0 & 0 & $34(94.4)$ & \\
\hline Parotid glands $(\mathrm{n}=31)$ & $3(9.7)$ & $1(3.2)$ & $4(12.9)$ & $23(74.2)$ & \\
\hline Hyperintensity artifacts & & & & & 0.729 \\
\hline Parotid lesions $(n=34)$ & $6(17.6)$ & $5(14.7)$ & $5(14.7)$ & $19(55.9)$ & \\
\hline Parotid glands ( $\mathrm{n}=28$ ) & $6(21.4)$ & $6(21.4)$ & $2(7.1)$ & $14(50.0)$ & \\
\hline
\end{tabular}



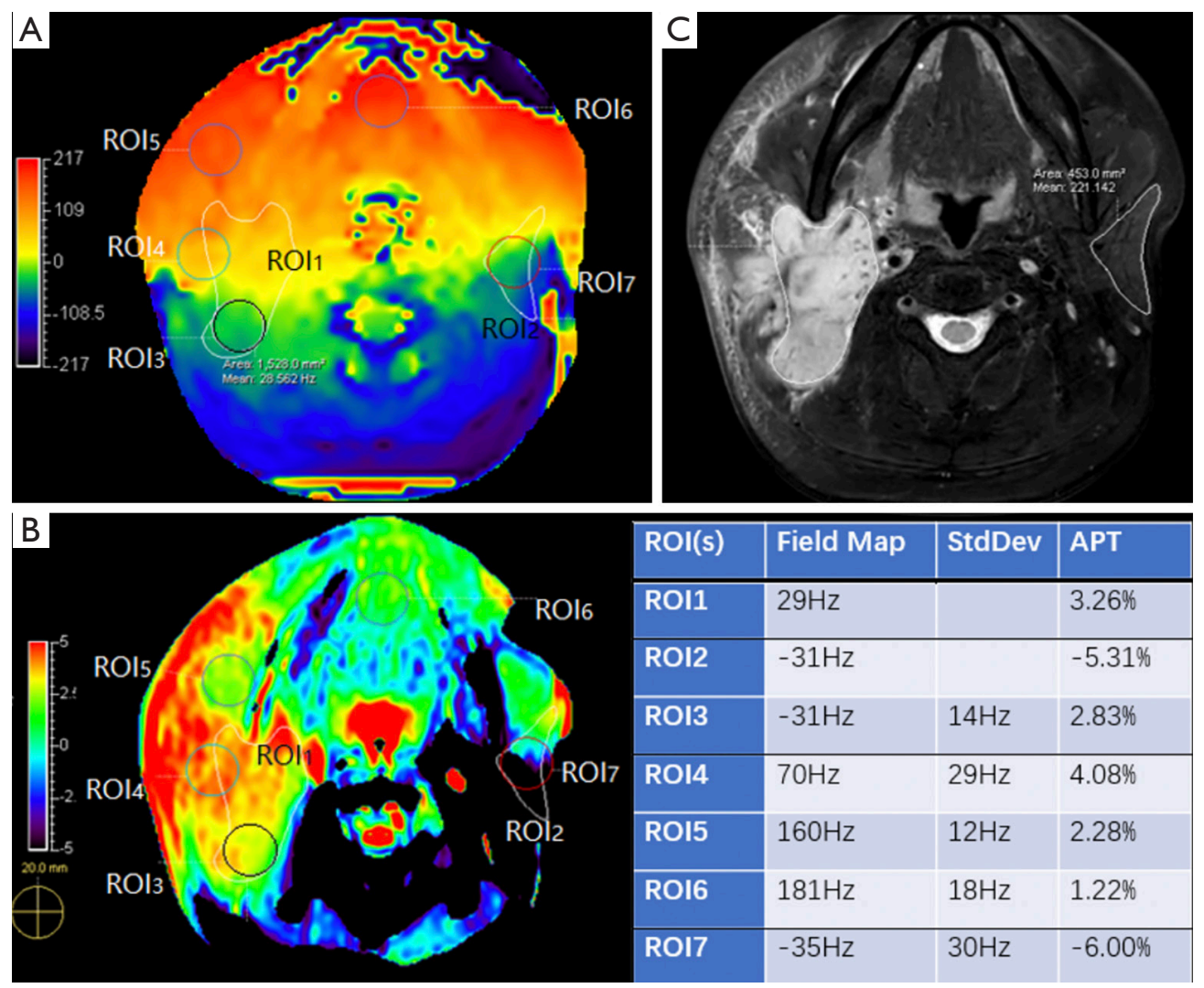

Figure 6 An example of the B0 map image (A) and APT image (B) on the parotid lesion and gland. A diffused lesion was found in the right

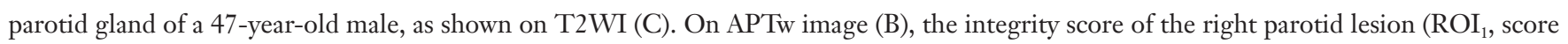
=4) was higher than that of the left normal parotid gland $\left(\mathrm{ROI}_{2}\right.$, score $\left.=1\right)$. Scores regarding the hyperintensity for the right parotid lesion and the left normal gland were 2 and 4, respectively. The parts with a higher standard deviation (StdDev) value $\left(\mathrm{ROI}_{4}\right.$ and $\left.\mathrm{ROI} 7\right)$ on the B0 map image had more APT artifacts (even hidden) compared to the ROI with a low StdDev value $\left(\mathrm{ROI}_{5}\right.$ and $\left.\mathrm{ROI}_{6}\right)$, though the mean value in the B0 was lower, indicating that a larger fluctuation in the B0 could be the key source of hyperintensity artifacts on APTw maps.

\section{Quantitative analysis}

After removing images with an integrity score of 1 , the average APTw value of 28 samples of parotid lesions and 22 parotid glands in the evaluable groups were $2.11 \% \pm 1.15 \%$ (range, $0.74-5.80 \%$ ) and $1.60 \% \pm 1.56 \%$ (range, $-0.96 \%$ to $4.53 \%)$, respectively. There were no significant differences $(\mathrm{P}=0.197)$ in the direct comparison of APTw values between parotid lesions and glands.

Next, after removing non-optimal image quality in lesions or parotid glands (integrity score $=2$ and 3 , and image quality scores $=1$ and 2), the average APTw value of 24 samples of parotid lesions and 14 parotid glands in the trustable groups were $1.99 \% \pm 1.18 \%$ (range, $0.74-5.80 \%$ ) and $1.03 \% \pm 1.09 \%$ (range, $-0.95 \%$ to $2.8 \%$ ), respectively. There was a notable difference between lesions and parotid glands $(\mathrm{P}=0.018)$. The quantitative analysis results are shown in Table 4.

\section{Discussion}

This preliminary study investigated the first feasibility test of 3D TSE APTw MR imaging for parotid gland tumors. There are some advantages to the 3D TSE APTw MR method compared to previously-published 2D APT methods. In addition to the easy implementation with in-line post-processing, the slice with the biggest tumor diameter can also be retrieved. However, there are artifacts in APTw images based on the current technique. Only trustable samples, which can be identified via a measurable method, should be enrolled in preliminary studies. Hence, we developed a strategy in this study to initially evaluate 
Table 4 Quantitative analysis of parotid lesions and normal parotid glands

\begin{tabular}{|c|c|c|c|}
\hline Projects & Parotid lesions & Parotid glands & $P$ value \\
\hline \multicolumn{4}{|l|}{ Evaluable groups } \\
\hline Patient numbers & 28 & 22 & \\
\hline APT value & $2.11 \% \pm 1.15 \%(0.74-5.80 \%)$ & $1.60 \% \pm 1.56 \%(-0.96 \%$ to $4.53 \%)$ & 0.197 \\
\hline \multicolumn{4}{|l|}{ Trustable groups } \\
\hline APT value & $1.99 \% \pm 1.18 \%(0.74-5.80 \%)$ & $1.03 \% \pm 1.09 \%(-0.95 \%$ to $2.8 \%)$ & 0.018 \\
\hline
\end{tabular}

Values given in parenthesis are the range of APT values.
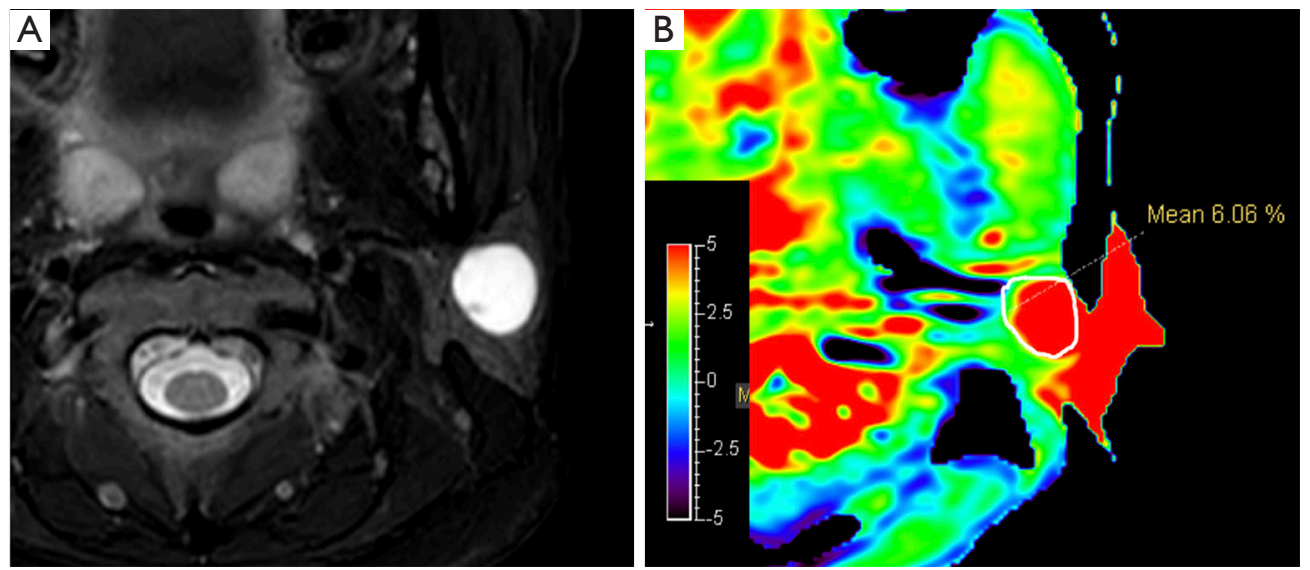

Figure 7 An APTw image example of a hyperintensity artifact caused by cysts. A hyperintensity-signal lesion was found in the left parotid gland of 39-year-old female, as shown on T2WI (A). The pathological result was pleomorphic adenoma with cystic change. On the APTw (B) image, the integrity score was 4 . The image quality in terms of hyperintensity artifacts scores was initially scored 4 by one radiologist and 2 by another. Eventually, the radiologists agreed on an image quality score of 2 because the hyperintensity in the cystic part of image was strongly affected by the artifacts diffused from the ear before the skull was hidden from the APT images.

the image quality in terms of integrity and hyperintensity artifacts and then selected APT image samples with good image quality to continue the feasibility test.

Our result showed that the APT values of parotid gland lesions were higher than those of normal parotid glands. This is consistent with APT findings in other anatomies, including the brain, prostate, and cervical cancers. Previous research has demonstrated that higher APT values indicate a higher cellular proliferation rate, and our application in parotid glands is consistent with this assumption.

There are a few types of image artifacts. Firstly, the images have a low-resolution appearance after a low pass filter, and the hyperintensity artifacts from the low-fidelity APTw values near bone and air diffuse to parotid glands, thereby potentially triggering false positives. Higher- resolution APT imaging would be desirable with the less smoothing effect so that lesions and parotid glands would be less affected by surrounding hyperintensities. Second, large areas of liquefied necrosis, hemorrhage, or even large blood vessels often present high APTw values (30), which might be due to excessive water-exchangeable amide protons (i.e., proteins and peptides in the blood, cytoplasm, or other body fluids), could also be influenced by nuclear Overhauser Effect (NOE) in asymmetry analysis causing a high APT signal in fluids (31).

In our study, parts of pleomorphic adenoma with cystic area presented hyperintensity artifacts (Figure 7). Furthermore, the normal parotid gland's intratumoral vessels always demonstrate hyperintensity on APTw images, as shown in the example in Figure 8. This finding 

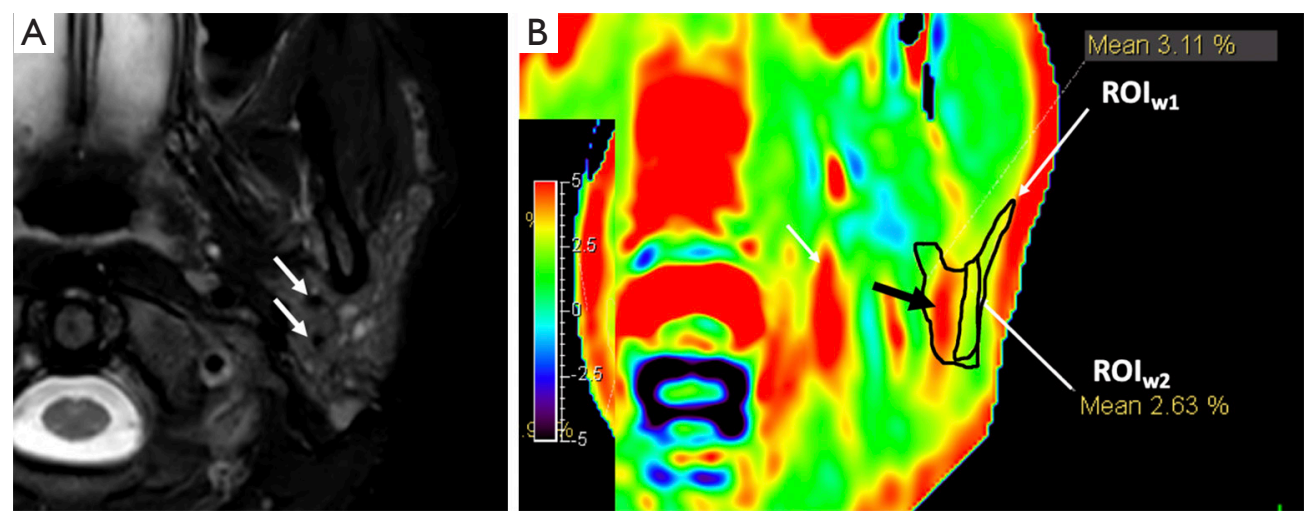

Figure 8 An APTw image example of a hyperintensity artifact caused by vessels. Image (A) T2WI shows the normal gland of the left side of a 28-year-old female with vessels passing through (white arrows). On the APTw (B) image, the APT image integrity was scored 4. The image quality in terms of hyperintensity artifacts was scored 3 because the APT values in the $\mathrm{ROI}_{\mathrm{w} 1}$ were moderately affected by the hyperintensity of the vessel (black arrow), while part of normal gland was unaffected (as in $\mathrm{ROI}_{\mathrm{w1}}$ ). The resulting mean APTw value of the parotid gland was $2.63 \%$ in $\mathrm{ROI}_{\mathrm{w} 2}$.

is consistent with Chen et al.'s study of the liver (32), where the intrahepatic portal vein system and hepatic arteries were found to have high APTw values. In a recent study by Jiang et al. (33), tumor vessels in low-grade gliomas were shown always to exhibit high values on APTw images. Liquefaction necrosis, cystic degeneration, or large blood vessels could be identified in conventional anatomical MRI images (such as $\mathrm{T} 2 \mathrm{w}$ and $\mathrm{T} 1 \mathrm{w}$ ); hence a reference to anatomical images is very useful to recognize APT image artifacts. These artifacts should be avoided when measuring APTw values for accurate interpretation (30). Thus, the image quality evaluation protocol is useful in screening for false positives and could serve as quality control.

The main challenge for APT imaging is B0 field inhomogeneity (20). Ideally, the APT calculation only needs to acquire images with saturation $\mathrm{RF}$ applied at $\pm 3.5 \mathrm{ppm}$. However, the applied RF tuned at fixed frequencies could not saturate exactly at $\pm 3.5 \mathrm{ppm}$ due to $\mathrm{B} 0$ variation, while magnetization transfer ratio (MTR) asymmetry analysis depends on correct z-spectrum measurement. To improve B0 homogeneity, linear (and even second-order shimming) is used; however, B0 is still affected by bone, air, and other susceptible factors $(11,24,34)$. Hence, a B0 map was measured (by acquiring three volumes with shifting echoes) to correct the z-spectrum with multiple offsets $(3.5 \mathrm{ppm}$, $3.5 \pm 0.8 \mathrm{ppm}$, and $-3.5 \mathrm{ppm},-3.5 \pm 0.8 \mathrm{ppm}$ ) acquired. To calculate the MTR asymmetry, the z-spectrum was shifted according to B0 at each voxel, and the measured points in the $\mathrm{z}$-spectrum were used to interpolate the correct value. Our results showed the z-spectrum shift algorithm provided the necessary correction to generate APTw maps of parotid gland tumors (Figure 9). However, the APT value is complicated by B0, which is problematic in near air-tissue interfaces, resulting in strong artifacts in the near ear and oral cavities. Some tumor lesions and normal parotid glands had low image quality due to the poor B0 homogeneity (Figure 6). For B0 inhomogeneity that was too large to shift the $\mathrm{z}$-spectrum within $0.8 \mathrm{ppm}$ offsets, extrapolation fitting was used (rather than interpolation), and unacceptable errors would occur due to lack of accuracy during extrapolation fitting. We also found that the region with stronger B0 variation, represented as a higher standard deviation in the B0 map, had more APT hyperintensity artifacts, indicating that a large fluctuation in the B0 map is the key source of residual artifacts on APTw maps. RF labeling of amide proton saturation is significantly important for an APT sequence, and the variations in RF strength would lead to errors in APT calculation. Hence, the homogeneity of B1 plays an important role in defining correct saturations. There had been developments in the correction of B1 inhomogeneity (35), and integration of the new method might improve the diagnostic value of APT results.

Another source of artifacts in the APT image is the negligence of the NOE effect. The NOE effect affects the MTR asymmetry (similar to the APT effect), and there are errors in the APTw value if $\mathrm{NOE}$ is ignored in the calculation. Zhou et al. (36) showed that NOE positively 


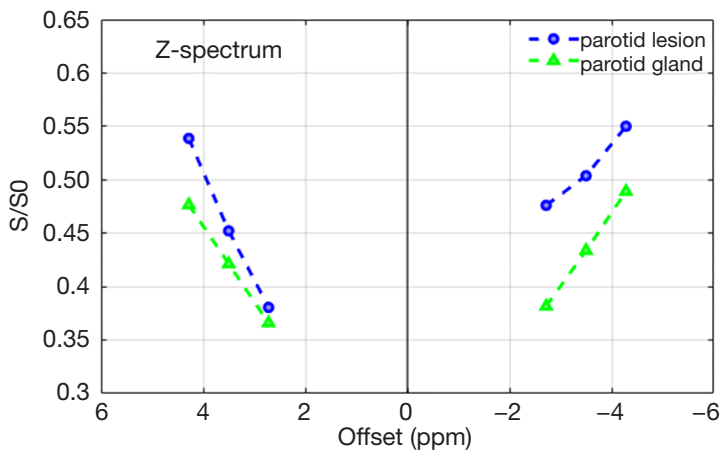

Figure 9 Measured $\mathrm{z}$-spectrums at $\pm 2.7 \mathrm{ppm}, \pm 3.5 \mathrm{ppm}$, and $\pm 4.3 \mathrm{ppm}$ (relative to water protons) normalized to signal S0 with far offset $(-1,560 \mathrm{ppm})$ saturation. The green curve and blue curves are the $z$-spectrum acquired from a parotid gland and a parotid lesion, respectively. The decreased signal from the parotid lesion at 3.5 ppm side corresponds to amide-water proton exchange.

affects APTw in tumors, and thus, our report on APTw value measurement in tumors could be overestimated, and NOE has contributed to some high APTw values.

The APT sequence's saturation RF pulses in the present study lasted for 2 seconds, with an amplitude of $2 \mu \mathrm{T}$, and the resulting APTw value corresponds to these particular B1 settings (continuous labeling time and strength). The APTw value will change if the saturation RF pulses are changed.

A study by Takeda et al. showed that the parotid gland's saliva contains amino acids, pyruvic acid, and lactic acid (37). In normal tissues studied by Yuan et al. (11), the parotid glands showed the highest APTw signal, while the APTw signals in other tissues (submandibular gland, thyroid gland, and masseter muscle) were relatively moderate. The APTw value was influenced by the amide concentration and the proton exchange ratio between amide and water. Hence, the APTw value is affected by the acidity of tissue (11). Future investigations need to address amide concentration measurement more directly, which might have a greater clinical impact on parotid gland lesion diagnosis.

Our study had several limitations in the final data interpretation. Firstly, not all of the parotid gland tumors had pathological results. Secondly, image data with poor image quality were ruled out for quantitative analysis, which reduced the total sampling size. Thirdly, although the image integrity could be objectively evaluated, the current image quality analysis protocol regarding hyperintensity artifacts requires radiologists experienced in parotid anatomy and APTw image reading. Fourthly, significant differences between parotid lesions and healthy glands were only observed after removing data with non-optimal image quality. Fifthly, parotid glands' contralateral sides without tumor lesions were considered the normal parotid gland groups. For these reasons, future prospective studies with a larger sample size are planned to characterize parotid gland tumors using the APT method. Lastly, we implemented the APTw protocol with a slice thickness of $5 \mathrm{~mm}$ to achieve a higher signal to noise ratio (SNR). However, the in-plane resolution and slice thickness restricted to use for smaller anatomy. Small lesions were not in the scope of this current preliminary study.

\section{Conclusions}

Our preliminary study of APT imaging for parotid tumors demonstrated that 3D APTw could be used to differentiate parotid tumors and normal parotid glands; however, the technology still needs to be improved to remove artifacts. Most APTw images of parotid glands' tumor lesions had acceptable image quality, and these APTw images are feasible for diagnostic use.

\section{Acknowledgments}

Funding: This work was funded by the National Natural Science Foundation of China (82001814), the National Public Welfare Basic Scientific Research Program of the Chinese Academy of Medical Sciences (2018PT32003 and 2017PT32004), and the Fundamental Research Funds for the Central Universities (3332018006).

\section{Footnote}

Conflicts of Interest: All authors have completed the ICMJE uniform disclosure form (available at http://dx.doi. org/10.21037/qims-20-675). The authors have no conflicts of interest to declare.

Ethical Statement: This study was conducted following the Declaration of Helsinki and was approved by an institutional review board. Written informed consent was obtained from the patients.

Open Access Statement: This is an Open Access article distributed in accordance with the Creative Commons Attribution-NonCommercial-NoDerivs 4.0 International License (CC BY-NC-ND 4.0), which permits the non- 
commercial replication and distribution of the article with the strict proviso that no changes or edits are made and the original work is properly cited (including links to both the formal publication through the relevant DOI and the license). See: https://creativecommons.org/licenses/by-nc-nd/4.0/.

\section{References}

1. Sumi M, Van Cauteren M, Sumi T, Obara M, Ichikawa Y, Nakamura T. Salivary gland tumors: use of intravoxel incoherent motion MR imaging for assessment of diffusion and perfusion for the differentiation of benign from malignant tumors. Radiology 2012;263:770-7.

2. Cho HW, Kim J, Choi J, Choi HS, Kim ES, Kim SH, Choi EC. Sonographically guided fine-needle aspiration biopsy of major salivary gland masses: a review of 245 cases. AJR Am J Roentgenol 2011;196:1160-3.

3. Lee YY, Wong KT, King AD, Ahuja AT. Imaging of salivary gland tumours. Eur J Radiol 2008;66:419-36.

4. Liu YJ, Lee YH, Chang HC, Chung HW, Wang CW, Juan CH, Chu YH, Lee JC, Juan CJ. Imaging quality of PROPELLER diffusion-weighted MR imaging and its diagnostic performance in distinguishing pleomorphic adenomas from Warthin tumors of the parotid gland. NMR Biomed 2020;33:e4282.

5. Sun Q, Ma C, Dong M, Jiang M, Tao X. Effects of region of interest sizes on apparent diffusion coefficient measurements of pleomorphic adenoma, Warthin tumor, and normal parotid parenchyma. Quant Imaging Med Surg 2019;9:681-90.

6. Yuan Y, Tang W, Tao X. Parotid gland lesions: separate and combined diagnostic value of conventional MRI, diffusionweighted imaging and dynamic contrast-enhanced MRI. Br J Radiol 2016;89:20150912.

7. Yabuuchi H, Kamitani T, Sagiyama K, Yamasaki Y, Hida T, Matsuura Y, Hino T, Murayama Y, Yasumatsu R, Yamamoto H. Characterization of parotid gland tumors: added value of permeability MR imaging to DWI and DCE-MRI. Eur Radiol 2020;30:6402-12.

8. Ohno Y, Yui M, Koyama H, Yoshikawa T, Seki S, Ueno Y, Miyazaki M, Ouyang C, Sugimura K. Chemical Exchange Saturation Transfer MR Imaging: Preliminary Results for Differentiation of Malignant and Benign Thoracic Lesions. Radiology 2016;279:578-89.

9. Wu B, Warnock G, Zaiss M, Lin C, Chen M, Zhou Z, Mu L, Nanz D, Tuura R, Delso G. An overview of CEST MRI for non-MR physicists. EJNMMI Phys 2016;3:19.

10. Dou W, Lin CE, Ding H, Shen Y, Dou C, Qian L, Wen
B, Wu B. Chemical exchange saturation transfer magnetic resonance imaging and its main and potential applications in pre-clinical and clinical studies. Quant Imaging Med Surg 2019;9:1747-66.

11. Yuan J, Chen S, King AD, Zhou J, Bhatia KS, Zhang Q, Yeung DK, Wei J, Mok GS, Wang YX. Amide proton transfer-weighted imaging of the head and neck at $3 \mathrm{~T}$ : a feasibility study on healthy human subjects and patients with head and neck cancer. NMR Biomed 2014;27:1239-47.

12. Mehrabian H, Myrehaug S, Soliman H, Sahgal A, Stanisz GJ. Evaluation of Glioblastoma Response to Therapy With Chemical Exchange Saturation Transfer. Int J Radiat Oncol Biol Phys 2018;101:713-23.

13. Regnery S, Adeberg S, Dreher C, Oberhollenzer J, Meissner JE, Goerke S, Windschuh J, Deike-Hofmann K, Bickelhaupt S, Zaiss M, Radbruch A, Bendszus M, Wick W, Unterberg A, Rieken S, Debus J, Bachert P, Ladd M, Schlemmer HP, Paech D. Chemical exchange saturation transfer MRI serves as predictor of early progression in glioblastoma patients. Oncotarget 2018;9:28772-83.

14. Dreher C, Oberhollenzer J, Meissner JE, Windschuh J, Schuenke P, Regnery S, Sahm F, Bickelhaupt S, Bendszus M, Wick W, Unterberg A, Zaiss M, Bachert P, Ladd ME, Schlemmer HP, Radbruch A, Paech D. Chemical exchange saturation transfer (CEST) signal intensity at 7T MRI of WHO IV gliomas is dependent on the anatomic location. J Magn Reson Imaging 2019;49:777-85.

15. Paech D, Dreher C, Regnery S, Meissner JE, Goerke S, Windschuh J, Oberhollenzer J, Schultheiss M, DeikeHofmann K, Bickelhaupt S, Radbruch A, Zaiss M, Unterberg A, Wick W, Bendszus M, Bachert P, Ladd ME, Schlemmer HP. Relaxation-compensated amide proton transfer (APT) MRI signal intensity is associated with survival and progression in high-grade glioma patients. Eur Radiol 2019;29:4957-67.

16. Dula AN, Arlinghaus LR, Dortch RD, Dewey BE, Whisenant JG, Ayers GD, Yankeelov TE, Smith SA. Amide proton transfer imaging of the breast at $3 \mathrm{~T}$ : establishing reproducibility and possible feasibility assessing chemotherapy response. Magn Reson Med 2013;70:216-24.

17. Dula AN, Dewey BE, Arlinghaus LR, Williams JM, Klomp D, Yankeelov TE, Smith S. Optimization of 7-T chemical exchange saturation transfer parameters for validation of glycosaminoglycan and amide proton transfer of fibroglandular breast tissue. Radiology 2015;275:255-61.

18. Donahue MJ, Donahue PC, Rane S, Thompson CR, Strother MK, Scott AO, Smith SA. Assessment of lymphatic impairment and interstitial protein 
accumulation in patients with breast cancer treatmentrelated lymphedema using CEST MRI. Magn Reson Med 2016;75:345-55.

19. Loi L, Zimmermann F, Goerke S, Korzowski A, Meissner JE, Deike-Hofmann K, Stieber A, Bachert P, Ladd ME, Schlemmer HP, Bickelhaupt S, Schott S, Paech D. Relaxation-compensated CEST (chemical exchange saturation transfer) imaging in breast cancer diagnostics at 7T. Eur J Radiol 2020;129:109068.

20. He YL, Li Y, Lin CY, Qi YF, Wang X, Zhou HL, Yang JJ, Xiang Y, Xue HD, Jin ZY. Three-dimensional turbospin-echo amide proton transfer-weighted mri for cervical cancer: A preliminary study. J Magn Reson Imaging 2019;50:1318-25.

21. Sun H, Xin J, Zhou J, Lu Z, Guo Q. Applying Amide Proton Transfer MR Imaging to Hybrid Brain PET/ MR: Concordance with Gadolinium Enhancement and Added Value to [18F]FDG PET. Mol Imaging Biol 2018;20:473-81.

22. Jia G, Abaza R, Williams JD, Zynger DL, Zhou J, Shah ZK, Patel M, Sammet S, Wei L, Bahnson RR, Knopp MV. Amide proton transfer MR imaging of prostate cancer: a preliminary study. J Magn Reson Imaging 2011;33:647-54.

23. Takayama $Y$, Nishie A, Sugimoto M, Togao O, Asayama Y, Ishigami K, Ushijima Y, Okamoto D, Fujita N, Yokomizo A, Keupp J, Honda H. Amide proton transfer (APT) magnetic resonance imaging of prostate cancer: comparison with Gleason scores. MAGMA 2016;29:671-9.

24. Bae YJ, Choi BS, Jeong WJ, Jung YH, Park JH, Sunwoo L, Jung C, Kim JH. Amide Proton Transfer-weighted MRI in the Diagnosis of Major Salivary Gland Tumors. Sci Rep 2019;9:8349.

25. Zhu H, Jones CK, van Zijl PC, Barker PB, Zhou J. Fast $3 \mathrm{D}$ chemical exchange saturation transfer (CEST) imaging of the human brain. Magn Reson Med 2010;64:638-44.

26. Yu L, Li C, Luo X, Zhou J, Zhang C, Zhang Y, Chen $M$. Differentiation of Malignant and Benign Head and Neck Tumors with Amide Proton Transfer-Weighted MR Imaging. Mol Imaging Biol 2019;21:348-55.

27. Eggers H, Brendel B, Duijndam A, Herigault G. Dualecho Dixon imaging with flexible choice of echo times. Magn Reson Med 2011;65:96-107.

28. Togao O, Keupp J, Hiwatashi A, Yamashita K, Kikuchi K, Yoneyama $\mathrm{M}$, Honda $\mathrm{H}$. Amide proton transfer imaging of brain tumors using a self-corrected 3D fast spin-echo dixon method: Comparison With separate B0 correction. Magn Reson Med 2017;77:2272-9.

29. Zhou J, Zhu H, Lim M, Blair L, Quinones-Hinojosa A,
Messina SA, Eberhart CG, Pomper MG, Laterra J, Barker PB, van Zijl PC, Blakeley JO. Three-dimensional amide proton transfer MR imaging of gliomas: Initial experience and comparison with gadolinium enhancement. J Magn Reson Imaging 2013;38:1119-28.

30. Zhou J, Heo HY, Knutsson L, van Zijl PCM, Jiang S. APT-weighted MRI: Techniques, current neuro applications, and challenging issues. J Magn Reson Imaging. 2019;50:347-64.

31. Paech D, Zaiss M, Meissner JE, Windschuh J, Wiestler B, Bachert P, Neumann JO, Kickingereder P, Schlemmer HP, Wick W, Nagel AM, Heiland S, Ladd ME, Bendszus M, Radbruch A. Nuclear overhauser enhancement mediated chemical exchange saturation transfer imaging at 7 Tesla in glioblastoma patients. PLoS One 2014;9:e104181.

32. Chen SZ, Yuan J, Deng M, Wei J, Zhou J, Wáng YX. Chemical exchange saturation transfer (CEST) MR technique for in-vivo liver imaging at 3.0 tesla. Eur Radiol 2016;26:1792-800.

33. Jiang S, Zou T, Eberhart CG, MAV V, Heo HY, Zhang Y, Wang Y, Wang X, Yu H, Du Y, van Zijl PCM, Wen Z, Zhou J. Predicting IDH mutation status in grade II gliomas using amide proton transfer-weighted (APTw) MRI. Magn Reson Med 2017;78:1100-9.

34. Law BKH, King AD, Ai QY, Poon DMC, Chen W, Bhatia KS, Ahuja AT, Ma BB, Ka-Wai Yeung D, Fai Mo FK, Wang YX, Yuan J. Head and Neck Tumors: Amide Proton Transfer MRI. Radiology 2018;288:782-90.

35. Windschuh J, Zaiss M, Meissner JE, Paech D, Radbruch A, Ladd ME, Bachert P. Correction of B1-inhomogeneities for relaxation-compensated CEST imaging at 7T. NMR Biomed 2015;28:529-37.

36. Zhou J, Hong X, Zhao X, Gao JH, Yuan J. APT-weighted and NOE-weighted image contrasts in glioma with different RF saturation powers based on magnetization transfer ratio asymmetry analyses. Magn Reson Med 2013;70:320-7.

37. Takeda I, Stretch C, Barnaby P, Bhatnager K, Rankin $\mathrm{K}, \mathrm{Fu} \mathrm{H}$, Weljie A, Jha N, Slupsky C. Understanding the human salivary metabolome. NMR Biomed 2009;22:577-84.

Cite this article as: Chen $\mathrm{Y}$, Wang $\mathrm{X}$, Su T, Xu Z, Wang $\mathrm{Y}$, Zhang Z, Xue H, Zhuo Z, Zhu Y, Jin Z, Zhang T. Feasibility evaluation of amide proton transfer-weighted imaging in the parotid glands: a strategy to recognize artifacts and measure APT value. Quant Imaging Med Surg 2021;11(6):2279-2291 doi: 10.21037/qims-20-675 